\title{
Quality of diet and mortality among Japanese men and women: Japan Public Health Center based prospective study
}

\author{
Kayo Kurotani,, Shamima Akter,, Ikuko Kashino, ${ }^{1}$ Atsushi Goto, ${ }^{2}$ Tetsuya Mizoue, \\ Mitsuhiko Noda, ${ }^{3,4}$ Shizuka Sasazuki, ${ }^{5}$ Norie Sawada, ${ }^{5}$ Shoichiro Tsugane'; Japan Public Health \\ Center based Prospective Study Group
}

'Department of Epidemiology and Prevention, Centre for Clinical Sciences, National Centre for Global Health and Medicine, 1-21-1 Toyama, Shinjuku-ku, Tokyo, Japan, 162-8655

${ }^{2}$ Department of Public Health, Tokyo Women's Medical University, 8-1 Kawadacho, Shinjuku-ku, Tokyo, Japan, 162-8666

32Department of Diabetes Research, National Centre for Global Health and Medicine, 1-21-1 Toyama, Shinjuku-ku, Tokyo, Japan, 162-8655

4Department of Endocrinology and Diabetes, Saitama Medical University, 38 Moro-hongo, Moroyama, Iruma-gun, Saitama, Japan, 350-0495

${ }^{5}$ Epidemiology and Prevention Group, Research Centre for Cancer Prevention and

Screening, National Cancer Centre, 5-1-1 Tsukiji, Chuo-ku, Tokyo, Japan, 104-0045

Correspondence to: K Kurotani kkurotani@ri.ncgm.go.jp

Additional material is published online only. To view please visit the journal online.

Cite this as: BMJ 2016;352:i1209 http://dx.doi.org/10.1136/bmj.i1209

Accepted: 12 Feb 2016

\section{ABSTRACT}

OBJECTIVE

To examine the association between adherence to the Japanese Food Guide Spinning Top and total and cause specific mortality.

DESIGN

Large scale population based prospective cohort study in Japan with follow-up for a median of 15 years.

SETTING

11 public health centre areas across Japan.

PARTICIPANTS

36624 men and 42970 women aged $45-75$ who had no history of cancer, stroke, ischaemic heart disease, or chronic liver disease.

\section{MAIN OUTCOME MEASURES}

Deaths and causes of death identified with the residential registry and death certificates.

\section{RESULTS}

Higher scores on the food guide (better adherence) were associated with lower total mortality; the multivariable adjusted hazard ratios ( $95 \%$ confidence interval) of total mortality for the lowest through highest scores were $1.00,0.92$ (0.87 to 0.97 ), 0.88 (0.83 to 0.93 ), and 0.85 ( 0.79 to 0.91$)(P<0.001$ for trend) and the multivariable adjusted hazard ratio associated with a 10 point increase in food guide scores was 0.93 (0.91 to 0.95; $\mathrm{P}<0.001$ for trend). This score was inversely associated with mortality from cardiovascular disease (hazard ratio associated with a 10 point increase $0.93,0.89$ to 0.98 ; $\mathrm{P}=0.005$ for trend) and particularly from cerebrovascular disease $(0.89,0.82$ to $0.95 ; \mathrm{P}=0.002$ for trend). There was some evidence, though not significant, of an inverse association for cancer mortality $(0.96,0.93$ to 1.00; $P=0.053$ for trend).

\section{CONCLUSION}

Closer adherence to Japanese dietary guidelines was associated with a lower risk of total mortality and mortality from cardiovascular disease, particularly from cerebrovascular disease, in Japanese adults.

\section{WHAT IS ALREADY KNOWN ON THIS TOPIC}

Studies in Western populations have shown that better quality of diet is associated with lower mortality from major chronic diseases

The evidence on this issue from prospective studies is scarce among Asian populations

\section{WHAT THIS STUDY ADDS}

Closer adherence to Japanese dietary guidelines was associated with a lower risk of death from all causes and cardiovascular disease, particularly cerebrovascular disease Balanced consumption of various dishes might contribute to longevity in the Japanese population

\section{Introduction}

Life expectancy of the Japanese population has steadily increased over the past few decades and is currently among the longest in the world, with Japanese women recording the longest life expectancy of 87 in $2012 .{ }^{1}$ Various factors, including socioeconomic and cultural background, as well as universal coverage of health insurance, have jointly contributed to the improvement of health among the Japanese population. ${ }^{2}$ Of these, special attention has been paid to the role of the Japanese diet, which has been improved in terms of nutritional balance during economic development ${ }^{34}$ while maintaining the traditional dietary culture (for instance, high intake of fish and soybean products and low intake of fat). ${ }^{5}$ Thus, the association between the diet of the Japanese population and risk mortality is of substantial clinical interest.

The assessment of overall diet quality has been shown to be useful in evaluating the effect of diet on morbidity or mortality. ${ }^{6}$ More than 25 a priori defined dietary scoring systems have been developed to assess diet quality based on adherence to dietary recommendations or guidelines specific to the country where the tool was developed. ${ }^{6}$ For example, the healthy eating index $^{7}$ and the alternate healthy eating index ${ }^{8}$ were developed in the United States according to Dietary Guidelines for Americans on the basis of nutrient and food intake. In Japan, the Ministry of Health, Labour and Welfare and the Ministry of Agriculture, Forestry and Fisheries of Japan jointly developed the Japanese Food Guide Spinning Top in 2005 (fig 1). ${ }^{9}$ This guide attempts to illustrate the balance and quantity of food in the daily Japanese diet. ${ }^{9}$ In 2009, Oba and colleagues developed a scoring system to measure the adherence to the food guide based on intake of grain dishes, vegetable dishes, fish and meat dishes, milk, and fruits, as well as the total daily energy and energy from snacks and alcoholic beverages. ${ }^{10}$

Higher diet quality scores have been consistently associated with a lower risk of mortality. ${ }^{10-25}$ All cause mortality was reportedly reduced by $11-42 \%,{ }^{10-25}$ cardiovascular disease mortality by 17-60\%, 10 11 14-16 18-20 22-24 and cancer mortality by 11-40\%11 14-16 181923 in individuals with the highest category of scores of diet quality compared with individuals with the lowest category. To the best of our knowledge, only two Asian studies have previously examined the association between diet quality and mortality. These studies showed an association between adherence to the Japanese Food Guide Spinning Top and the Chinese Food Pagoda and lower risk of mortality in each of the respective populations. ${ }^{1019}$ As the Japanese study was conducted in 


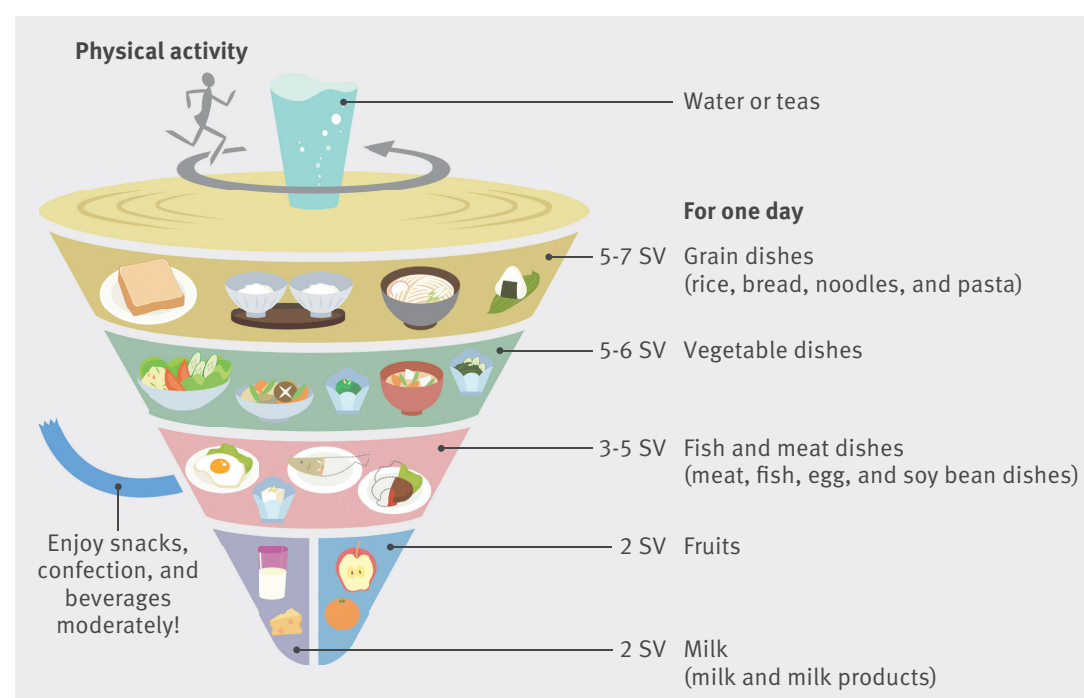

SV is an abbreviation of "serving" which is a simply countable number describing estimated amount of each dish or food served to one person determined by Ministry of Health, Labour and Welfare and Ministry of Agriculture, Forestry and Fisheries

Fig 1 | Japanese Food Guide Spinning Top

\section{Food frequency questionnaire}

The second survey included data on 147 food and beverage items and nine frequency categories. ${ }^{28}$ For most food items, nine options were available for describing consumption frequency, ranging from rarely (less than once a month) to seven or more times a day. Regarding food intake, a standard portion size was specified for each food item and the respondents were asked to assess their usual portion size relative to the standard specified for each food item using three options (less than half, standard, and more than one and half times). Daily intake of most foods was calculated by multiplying the daily consumption frequency and the standard portion size by the individual's usual portion size. From the food frequency questionnaire, we estimated total daily energy intake and intake of each nutrient and food item according to the Japanese standard tables of food composition. ${ }^{29}$ The food frequency questionnaire has reasonable validity and reproducibility. ${ }^{30-32}$ As regards validity, the Spearman rank correlation coefficients for energy adjusted intakes of food groups between diet records and the food frequency questionnaire ranged from 0.08 for algae to 0.76 for alcoholic beverages in men and 0.06 for algae to 0.66 for milk and dairy products in women in cohort $\mathrm{I}^{32}$ and from 0.11 for algae to 0.69 for milk and dairy products in men and 0.12 for fungi to 0.65 for pickled vegetables in women in cohort II. ${ }^{30}$ As regard reproducibility, the Spearman rank correlation coefficients for energy adjusted intakes of food groups measured at 12 month intervals with the food frequency questionnaire ranged from 0.38 for total beverages to 0.71 for alcoholic beverages in men and 0.30 for nuts and seeds to 0.74 for milk and dairy products in women in cohort $\mathrm{I}^{31}$ and from 0.38 for algae to 0.70 for alcoholic beverages in men and 0.40 for fish and shellfish and algae to 0.80 for alcoholic beverages in women in cohort II. ${ }^{30}$

\section{Standard and modified scores on Japanese food guide}

We created a score for adherence to a Japanese food guide, with a higher score indicating closer adherence and thus better diet. The Japanese Food Guide Spinning Top is based on the Dietary Guidelines for Japanese, released in 2000, and guides people as to the types and amounts of food they should eat each day to promote health. The food guide comprises five categories of dish: grain dishes (including rice, bread, and noodles), vegetable dishes (including vegetables, mushrooms, potatoes, and seaweed), fish and meat dishes (including meat, fish, eggs, and soybeans, milk (milk and milk products), and fruits (fruit and 100\% fruit juice) (fig 1). ${ }^{9}$ Soybeans are included in category of fish and meat dishes on the basis of nutrient profile (high protein). In the food guide, the amount of food that counts as one serving is defined for each category. One serving of a grain dish is composed of about $40 \mathrm{~g}$ carbohydrates. In one serving of a vegetable dish, the main ingredient weighs about $70 \mathrm{~g}$. One serving of fish and meat dish contains about $6 \mathrm{~g}$ protein, and one serving of milk contains about $100 \mathrm{mg}$ calcium. In one serving of fruits, the 


\begin{tabular}{|c|c|c|c|c|c|c|c|}
\hline Age and level of physical activity & $\begin{array}{l}\text { Grain dishes } \\
\text { (serving/d) }\end{array}$ & $\begin{array}{l}\text { Vegetable } \\
\text { dishes* } \\
\text { (serving/d) }\end{array}$ & $\begin{array}{l}\text { Fish and } \\
\text { meat dishes } \\
\text { (serving/d) }\end{array}$ & $\begin{array}{l}\text { Milk } \\
\text { (serving/d) }\end{array}$ & $\begin{array}{l}\text { Fruits* } \\
\text { (serving/d) }\end{array}$ & $\begin{array}{l}\text { Total energy } \\
\text { (kcal/d) }\end{array}$ & $\begin{array}{l}\text { Snacks and } \\
\text { alcoholic } \\
\text { beverages } \\
(\mathrm{kcal} / \mathrm{d})\end{array}$ \\
\hline Men aged $\geq 70$; women aged $\geq 70$ or sedentary women aged 18-69 & 4-5 & $5-6$ & $3-4$ & 2 & 2 & $1600-2000$ & $0-200$ \\
\hline Sedentary men aged 18-69; moderately active women aged 18-69 & $5-7$ & $5-6$ & $3-5$ & 2 & 2 & $2000-2400$ & $0-200$ \\
\hline Moderately active men aged 18-69 & $7-8$ & $6-7$ & $4-6$ & $2-3$ & $2-3$ & $2400-2800$ & $0-200$ \\
\hline
\end{tabular}

main ingredient weighs about $100 \mathrm{~g}$. The recommended amount of servings for each category and the recommended total energy intake are specified according to sex, age, and level of physical activity, whereas the recommended amount of energy intake from snacks and alcoholic beverages is less than $200 \mathrm{kcal} /$ day for everyone (table 1).

We determined the scores by measuring adherence to the food guide from information in the food frequency questionnaire. First, we calculated the number of servings according to the food guide criteria. Grain dishes included rice, vitamin enriched rice, rice with cereal, bread, Japanese wheat noodles, buckwheat noodles, Okinawa noodles, Chinese noodles, and rice cakes. Vegetable dishes included carrots, spinach, pumpkins, cabbage, Chinese cabbage, Chinese radishes, salted pickles of Chinese radishes, salted pickles of green leafy vegetables, pickled plums, pickled Chinese cabbage, pickled cucumbers, pickled eggplant, sweet pepper, tomatoes, Chinese chives, garland chrysanthemums, komatsuna, broccoli, onions, cucumbers, bean sprouts, snap beans, lettuce, pak choy, leaf mustard, bitter gourds, leaf beet, loofah, mugwort, sweet potato, potato, taro, shiitake mushroom, hackberry, wakame seaweed, dark edible seaweed, lavers, peanuts, and tomato juice. Fish and meat dishes included steak, grilled and stewed beef, stir fried pork, deep fried pork, Western style stewed pork, Japanese style stewed pork, pork in soup, pork liver, ham, sausage or Wiener sausage, bacon and luncheon meats, chicken liver, grilled chicken, deep fried chicken, egg, salmon, skipjack/tuna, cod/flatfish, sea bream, horse mackerel/sardines, saury/mackerel, eel, squid, octopus, shrimp, clams, pond snails, salted fish, dried fish, dried whitebait, salted fish roe, canned tuna, fish paste products (chikuwa and kamaboko), tofu, boiled tofu, fluffy tofu, freeze dried tofu, deep fried tofu, fermented soybean (natto), and soymilk (tofu and soy products are included in this category because of their nutrient profile). Milk included cows' milk, cheese, yoghurt, Japanese probiotic milk drink, and milk in tea and coffee. Fruits included papaya, mandarin oranges, other oranges, apples, persimmons, strawberries, grapes, melons, watermelon, peaches, pears, kiwifruit, pineapple, bananas, 100\% orange juice, and $100 \%$ apple juice. Snacks and alcoholic beverages included Japanese rice wine (sake), shochu, beer, whisky, wine, Japanese confectionaries, cake, biscuits, chocolate, canned coffee, coke, and sugar in tea and coffee.
Table 2 shows the procedure of creating an adherence score for the food guide. We classified participants who engaged in manual labour or walked at least one hour a day as moderately physically active and the remaining as sedentary. For vegetable dishes and fruits, we modified the original criteria (recommended range) to remove the upper limit of intake, conforming to other scoring systems. ${ }^{1621}$ For example, the food guide recommends five to six servings of vegetable dishes for men aged $\geq 70$, but we modified this to five or more servings. If individuals consumed the recommended amount of servings from any of the five categories or the recommended total energy or energy from snacks and alcoholic beverages, 10 points were given for that group. If individuals exceeded or fell short of the recommended servings or energy, the score was calculated proportionately between 0 and 10. If an individual consumed less than the recommended amount of servings or energy, the score was calculated with the following formula: $10 \times$ (the consumed amount of servings or energy)/(the lower limit of the recommended amount). If an individual consumed more than the recommended amount of servings or energy, the score was calculated with the following formula: 10-10 $\times[$ (the consumed amount of servings or energy)-(the upper limit of the recommended amount)]/(the upper limit of the recommended amount). Each score was rounded off to the nearest whole number. When this calculation produced a negative score because of excess servings or energy, the score was converted to 0 . All group scores were summed to obtain a total score ranging from 0 (the lowest adherence) to 70 (the highest adherence). We added the ratio of white to red meat as a new component to the score, creating the modified score according to the alternate healthy eating index. ${ }^{8}$ White meat was defined as grilled chicken, deep fried chicken, salmon, skipjack/ tuna, cod/flatfish, sea bream, horse mackerel/sardine, saury/mackerel, eel, squid, octopus, shrimp, clam, pond snails, salted fish, dried fish, dried whitebait, salted fish roe, canned tuna, and fish paste products. Red meat was defined as steak, grilled and stewed beef, stir fried pork, deep fried pork, Western style stewed pork, Japanese style stewed pork, pork in soup, pork liver, ham, sausage or Wiener sausage, bacon and luncheon meats, and chicken liver. Gram quantities were summed and used to calculate the ratio. An ideal score of 10 was given for ratios $\geq 4: 1,{ }^{8}$ whereas a score of 0 was given for a ratio of 0 . Intermediate intakes were scored proportionately between 0 and 10 . The food guide score and the ratio of white to red meat score were summed to 
Table 2 | Operationalisation of Japanese food guide score (scored components comprised grain dishes, vegetable dishes, fish and meat dishes, milk, fruits, total energy, and snacks and alcoholic beverages; total score 0-70) and modified score (scored components as for standard score plus ratio of white to red meat*; total score $0-80$ )

\begin{tabular}{|c|c|c|c|}
\hline Japanese Food Guide Spinning Top component and population & $\begin{array}{l}\text { Standard for } \\
\text { minimum score } \\
\text { of } 0 \text { points }\end{array}$ & $\begin{array}{l}\text { Standard for } \\
\text { continuous scoring } \\
\text { of } 0-10 \text { pointst }\end{array}$ & $\begin{array}{l}\text { Standard for } \\
\text { maximum score } \\
\text { of } 10 \text { points }\end{array}$ \\
\hline \multicolumn{4}{|l|}{ Grain dishes (serving/d)‡ } \\
\hline Men aged $\geq 70$; women aged $\geq 70$ or sedentary women aged $18-69$ & 0 or $\geq 10$ & $>0-<4$ or $>5-<10$ & 4-5 \\
\hline Sedentary men aged 18-69; moderately active women aged 18-69 & 0 or $\geq 14$ & $>0-<5$ or $>7-<14$ & $5-7$ \\
\hline Moderately active men aged 18-69 & 0 or $\geq 16$ & $>0-<7$ or $>8-<16$ & $7-8$ \\
\hline \multicolumn{4}{|l|}{ Vegetable dishes (serving/d)§ } \\
\hline Men aged $\geq 70$; women aged $\geq 70$ or sedentary women aged $18-69$ & 0 & $>0-<5$ & $\geq 5$ \\
\hline Sedentary men aged 18-69; moderately active women aged 18-69 & 0 & $>0-<5$ & $\geq 5$ \\
\hline Moderately active men aged 18-69 & 0 & $>0-<6$ & $\geq 6$ \\
\hline \multicolumn{4}{|l|}{ Fish and meat dishes (serving/d)ף } \\
\hline Men aged $\geq 70$; women aged $\geq 70$ or sedentary women aged $18-69$ & 0 or $\geq 8$ & $>0-<3$ or $>4-<8$ & $3-4$ \\
\hline Sedentary men aged 18-69; moderately active women aged 18-69 & 0 or $\geq 10$ & $>0-<3$ or $>5-<10$ & $3-5$ \\
\hline Moderately active men aged 18-69 & 0 or $\geq 12$ & $>0-<4$ or $>6-<12$ & $4-6$ \\
\hline \multicolumn{4}{|l|}{ Milk (serving/d)** } \\
\hline Men aged $\geq 70$; women aged $\geq 70$ or sedentary women aged $18-69$ & 0 or $\geq 4$ & $>0-<2$ or $>2-<4$ & 2 \\
\hline Sedentary men aged 18-69; moderately active women aged 18-69 & 0 or $\geq 4$ & $>0-<2$ or $>2-<4$ & 2 \\
\hline Moderately active men aged $18-69$ & 0 or $\geq 6$ & $>0-<2$ or $>3-<6$ & $2-3$ \\
\hline \multicolumn{4}{|l|}{ Fruits (serving/d)tt } \\
\hline Men aged $\geq 70$; women aged $\geq 70$ or sedentary women aged $18-69$ & 0 & $>0-<2$ & $\geq 2$ \\
\hline Sedentary men aged 18-69; moderately active women aged 18-69 & 0 & $>0-<2$ & $\geq 2$ \\
\hline Moderately active men aged $18-69$ & 0 & $>0-<2$ & $\geq 2$ \\
\hline \multicolumn{4}{|l|}{ Total energy $(\mathrm{kcal} / \mathrm{d})$} \\
\hline Men aged $\geq 70$; women aged $\geq 70$ or sedentary women aged $18-69$ & $\geq 4000$ & $<1600$ or $>2000-<4000$ & $1600-2000$ \\
\hline Sedentary men aged 18-69; moderately active women aged 18-69 & $\geq 4800$ & $<2000$ or $>2400-<4800$ & $2000-2400$ \\
\hline Moderately active men aged 18-69 & $\geq 5600$ & $<2400$ or $>2800-<5600$ & $2400-2800$ \\
\hline \multicolumn{4}{|l|}{ Snacks and alcoholic beverages (kcal/d) } \\
\hline Men aged $\geq 70$; women aged $\geq 70$ or sedentary women aged $18-69$ & $\geq 400$ & $>200-<400$ & $0-200$ \\
\hline Sedentary men aged 18-69; moderately active women aged 18-69 & $\geq 400$ & $>200-<400$ & $0-200$ \\
\hline Moderately active men aged $18-69$ & $\geq 400$ & $>200-<400$ & $0-200$ \\
\hline \multicolumn{4}{|c|}{$\begin{array}{l}\text { *White meat defined as poultry and fish/shellfish; red meat defined as beef, pork, and liver. Gram quantities summed and used to calculate ratio: ratio of } 0 \text { was standard for minimum score of } 0 \\
\text { points; ratio } \geq 4: 1 \text { was standard for maximum score of } 10 \text { points. } \\
\text { tIf individual consumed less than recommended amount of servings or energy, score was calculated with formula: } 10 \times \text { ( consumed amount of servings or energy)/( lower limit of recommended } \\
\text { amount). If individual consumed more than recommended amount of servings or energy, score was calculated with formula: 10-10x[( consumed amount of servings or energy)-( upper limit of } \\
\text { recommended amount)]/( upper limit of the recommended amount). } \\
\text { fIncludes rice, bread, and noodles. One serving contains about } 40 \mathrm{~g} \text { carbohydrates. } \\
\text { SIncludes vegetables, mushrooms, potatoes, and seaweed. In one serving, main ingredient weighs about } 70 \mathrm{~g} \text {. } \\
\text { IIncludes meat, fish, eggs, and soybeans. One serving contains about } 6 \mathrm{~g} \text { protein. } \\
\star * \text { Includes milk and milk products. One serving contains about } 100 \mathrm{mg} \text { calcium. } \\
+† \text { In one serving, main ingredient weighs about } 100 \mathrm{~g} \text {. }\end{array}$} \\
\hline
\end{tabular}

obtain a modified score ranging from 0 (worst) to 80 (best) (table 2).

\section{Study population}

Of the potential participants at baseline ( $\mathrm{n}=140420)$, 103450 responded to the second survey; of these, 102364 completed the food frequency questionnaire at the second survey. Of these participants, we excluded 10227 because of missing information regarding the number of bowls of rice consumed and the frequency of intake of the following: more than half of the vegetable items, more than half of the fish and meat dishes items, milk, more than half of the fruit items, all snack items, and alcoholic beverage items. Of 92137 participants, we excluded 6215 who reported consumption in the upper $1 \%$ of sex specific intakes of each category (grain dishes, vegetable dishes, fish and meat dishes, milk, fruits, as well as energy from snacks and alcohol) or in either the upper or lower $1 \%$ of sex specific energy intake. We also excluded 6328 participants who reported history of cancer, stroke, ischaemic heart disease, or chronic liver disease in the baseline and second survey. Ultimately, 79594 participants (36624 men and 42970 women) remained for the present analysis.

\section{Reproducibility of dietary intake}

Of the participants in the analytic cohort, 43676 (19778 men and 23898 women) participated in the 10 year follow-up survey (that is, the third survey) and completed the food frequency questionnaire. Dietary intake between the second and the third surveys was moderately stable. The Spearman rank correlation coefficients for each intake between the two surveys were as follows: 0.47 and 0.45 for grain dishes, 0.52 and 0.55 for vegetable dishes, 0.46 and 0.50 for fish and meat dishes, 0.52 and 0.51 for milk, 0.54 and 0.56 for fruits, 0.49 and 0.51 for total energy, 0.52 and 0.50 for energy from snacks, and 0.64 and 0.64 for alcohol in men and women, respectively. The score on the food guide between two surveys was also moderately stable. 
The Spearman rank correlation coefficient of the score was 0.50 in men and 0.45 in women.

\section{Follow-up and outcome}

We followed up the participants' residency and vital status from the second survey to 31 December 2012 using the residential registry. Causes of deaths were confirmed through death certificates (with permission) and were defined according to ICD-10 (international classification of diseases, 10th revision). ${ }^{33}$ The major endpoint of the present study was mortality from all causes, cancer (ICD-10 codes C00 to C97), cardiovascular disease (I00 to I99), heart disease (I20 to I52), and cerebrovascular disease (I60 to I69).

\section{Statistical analysis}

We calculated person years of follow-up for each person starting from the date of response to the second survey questionnaire until the date of death, emigration from Japan, or 31 December 2012, whichever came first. For individuals who were lost to follow-up, we used the last confirmed date of their participation in the study area as the censoring date. Participants were divided into fourths of food guide scores. We considered the following confounding variables collected at the second survey: age (years, continuous), sex, study area (11 areas), body mass index (BMI <21, 21.0-22.9, 23.0-24.9, 25.0-26.9, or $\geq 27.0$ ), smoking status (lifetime non-smoker, former smoker, or current smoker with a consumption of either $<20$ or $\geq 20$ cigarettes/day), total physical activity (metabolic equivalent task h/day, fourths), a history of diabetes mellitus (yes or no), a history of hypertension (yes or no), a history of dyslipidaemia (yes or no), coffee consumption (almost never, $<1$, 1 , or $\geq 2$ cups/day; 1 cup $=120$ $\mathrm{mL}$ ), green tea consumption (almost never, $<1,1,2-3$, or $\geq 4$ cups/day), and occupation (agriculture/forestry/ fishery, salaried/professional, self employed, multiple occupations, housework/unemployed, or other). For participants with missing data on BMI, smoking status, physical activity, coffee consumption, green tea consumption, or occupation, we imputed the data using multivariate normal imputation (the SAS PROC MI procedure) with five rounds of multiple imputations by including all covariates, follow-up length, and mortality status to account for missing data. We then combined the estimates from each imputed dataset using Rubin's rules (the SAS PROC MIANALYZE procedure). ${ }^{34}$ We used Cox proportional hazards regression analysis to estimate hazard ratios and 95\% confidence intervals of mortality from total and cause specific mortality for fourths of the food guide scores and the modified scores, using the lowest fourth as a reference. The first model was adjusted for age, sex, and study area, and the second model was further adjusted for BMI, smoking status, total physical activity, history of diabetes mellitus, history of hypertension, history of dyslipidaemia, coffee consumption, green tea consumption, and occupation. We estimated hazard ratios and the corresponding 95\% confidence intervals for a 10 point increase in the standard and modified scores. The linear trend was computed by treating exposure as a continuous variable. We confirmed that the quadratic term of the score added to the multivariable adjusted model was not significantly associated with any outcome. In addition, to visually inspect possible non-linear associations, we used restricted cubic spline with five knots placed at the 5th, 25th, 50th, 75th, and 95th centiles of the food guide score. The reference value for estimating hazard ratios and $95 \%$ confidence intervals was chosen as 8 , the lowest value of this score.

We estimated hazard ratios and the corresponding 95\% confidence intervals for mortality from total and cause specific mortality for a 1 point increment in the score for each dish category. To estimate the impact of each component of the score on the association between the total dietary score and mortality, we calculated the proportion of hazard ratio attenuation in the highest fourth with the following formula: (hazard ratio adjusted for all covariates plus each dish score-hazard ratio adjusted for all covariates [not including dish score])/(1-hazard ratio adjusted for all covariates [not including dish score]) $\times 100$.

Data were analysed stratified by sex, BMI $(<25$ or $\geq 25$ ), smoking status (men only), and menopausal status. By multiplying the score (continuous) and the above stratifying variables (dichotomous), we created an interaction term that we added to the model to assess statistical interactions. We conducted multiple sensitivity analyses by excluding individuals with a history of diabetes mellitus, hypertension, or dyslipidaemia and those who died during the first three years of follow-up. The proportional hazards assumption was tested by including a product term between fourths of the score and the follow-up period in the models; no significant violation of the assumption was found (all $\mathrm{P}>0.10$ ). All analyses were performed with SAS version 9.3 for Windows (SAS Institute, Cary, NC) and Stata version 13.1 (StataCorp, College Station, TX).

\section{Patient involvement}

No patients were involved in setting the research question or the outcome measures, nor were they involved in the design and implementation of the study. There are no plans to involve patients in dissemination.

\section{Results}

At baseline (the time of the second survey), the mean standard and modified scores on the Japanese Food Guide Spinning Top were 47.4 (SD 10.2) and 53.0 (SD 11.0), respectively. Scores can range from 0 to 70 (modified 0-80), with higher scores indicating better adherence and therefore a better diet. Compared with individuals with a lower dietary score, individuals with a higher score were older and more likely to be women, be engaged in primary industry, have a history of dyslipidaemia, drink green tea, and have a higher energy intake but were less likely to be a current smoker, drink alcohol every week, or have a history of hypertension (table 3).

\section{Main analyses}

During the mean follow-up time of 14.9 (SD 3.3) years, the numbers of deaths from all causes, cancer, 


\begin{tabular}{|c|c|c|c|c|}
\hline & \multicolumn{4}{|c|}{ Fourths of score } \\
\hline & $1(\mathrm{n}=20851)$ & $2(n=17708)$ & $3(n=21886)$ & $4(n=19149)$ \\
\hline Score (point) & $34.2(4.9)$ & $44.1(2.0)$ & $51.5(2.3)$ & $60.3(3.3)$ \\
\hline Age (years) & $50.4(7.7)$ & $51.0(7.8)$ & $51.2(7.7)$ & $51.5(7.5)$ \\
\hline Men (\%)† & 84.0 & 57.4 & 31.9 & 10.2 \\
\hline Mean (SE) BMI† & $23.5(0.02)$ & $23.5(0.02)$ & $23.5(0.02)$ & $23.4(0.02)$ \\
\hline Mean (SE) total physical activity (metabolic equivalents $\mathrm{h} /$ day) $\dagger$ & $33.3(0.05)$ & $33.1(0.05)$ & $32.9(0.05)$ & $33.0(0.05)$ \\
\hline Current smoker (\%)‡ & 24.3 & 18.3 & 14.9 & 10.5 \\
\hline Alcohol consumption ( $\geq 1$ day/week, \%) $\ddagger$ & 53.4 & 33.9 & 25.9 & 22.7 \\
\hline History of hypertension (\%) $\ddagger$ & 17.6 & 16.3 & 16.0 & 15.6 \\
\hline History of diabetes $(\%) \neq$ & 5.6 & 5.8 & 5.8 & 5.2 \\
\hline History of dyslipidaemia (\%) $\ddagger$ & 3.8 & 4.4 & 4.9 & 5.5 \\
\hline Mean (SE) energy intake $(\mathrm{kcal} / \mathrm{d}) \ddagger$ & $1872(4.4)$ & $1964(4.4)$ & $2047(4.0)$ & $2144(4.5)$ \\
\hline Coffee consumption ( $\geq 1$ cups/day, \%) $\ddagger$ & 35.1 & 36.7 & 36.6 & 33.4 \\
\hline Green tea consumption ( $\geq 1$ cups/day, \%) $\ddagger$ & 48.4 & 56.2 & 62.2 & 69.6 \\
\hline Occupation (agriculture, forestry, fishery, \%)‡ & 15.2 & 16.5 & 17.4 & 18.3 \\
\hline
\end{tabular}

\section{Table 4 | Multivariable adjusted hazard ratios (HR) and 95\% confidence intervals of mortality according to score on Japanese food guide (lower} score=lower adherence=lower quality diet)

\begin{tabular}{|c|c|c|c|c|c|c|}
\hline & \multicolumn{4}{|c|}{ Fourths of score } & \multirow[b]{2}{*}{10 point increment } & \multirow[b]{2}{*}{ P trend* } \\
\hline & 1 & 2 & 3 & 4 & & \\
\hline Mean (SD) score & $34.2(4.9)$ & $44.1(2.0)$ & $51.5(2.3)$ & $60.3(3.3)$ & - & - \\
\hline Person years & 301776 & 261794 & 328105 & 292520 & - & - \\
\hline \multicolumn{7}{|l|}{ Total mortality } \\
\hline No of deaths & 3497 & 2463 & 2470 & 1753 & - & - \\
\hline Rate/1000 person years & 11.6 & 9.4 & 7.5 & 6.0 & - & - \\
\hline Age, sex, and area adjusted HR $(95 \% \mathrm{CI}) \dagger$ & 1.00 (ref) & $0.86(0.82$ to 0.91$)$ & $0.80(0.75$ to 0.84$)$ & $0.74(0.70$ to 0.80$)$ & 0.88 (0.86 to 0.90$)$ & $<0.001$ \\
\hline Multivariable adjusted HR $(95 \% \mathrm{Cl}) \neq$ & 1.00 (ref) & 0.92 (0.87 to 0.97$)$ & 0.88 (0.83 to 0.93$)$ & 0.85 (0.79 to 0.91$)$ & 0.93 (0.91 to 0.95) & $<0.001$ \\
\hline \multicolumn{7}{|l|}{ Cancer mortality } \\
\hline No of deaths & 1421 & 995 & 1016 & 755 & - & - \\
\hline Rate/1000 person years & 4.7 & 3.8 & 3.1 & 2.6 & - & - \\
\hline Age, sex, and area adjusted HR $(95 \% \mathrm{CI}) \dagger$ & 1.00 (ref) & $0.89(0.82$ to 0.96$)$ & 0.85 (0.78 to 0.93) & $0.86(0.77$ to 0.96$)$ & 0.92 (0.89 to 0.95$)$ & $<0.001$ \\
\hline Multivariable adjusted HR $(95 \% \mathrm{Cl}) \neq$ & 1.00 (ref) & 0.94 (0.87 to 1.02$)$ & 0.94 (0.86 to 1.02$)$ & $0.96(0.87$ to 1.07$)$ & 0.96 (0.93 to 1.00$)$ & 0.053 \\
\hline \multicolumn{7}{|l|}{ Cardiovascular disease mortality } \\
\hline No of deaths & 843 & 640 & 619 & 458 & - & - \\
\hline Rate/1000 person years & 2.8 & 2.4 & 1.9 & 1.6 & - & - \\
\hline Age, sex, and area adjusted HR $(95 \% \mathrm{Cl}) \dagger$ & 1.00 (ref) & 0.89 (0.80 to 0.99$)$ & $0.77(0.69$ to 0.86$)$ & $0.72(0.63$ to 0.82$)$ & 0.87 (0.83 to 0.91$)$ & $<0.001$ \\
\hline Multivariable adjusted HR $(95 \% \mathrm{Cl}) \neq$ & 1.00 (ref) & $0.97(0.87$ to 1.07$)$ & $0.86(0.77$ to 0.97$)$ & 0.84 (0.73 to 0.96$)$ & 0.93 (0.89 to 0.98) & 0.005 \\
\hline \multicolumn{7}{|l|}{ Heart disease mortality } \\
\hline No of deaths & 445 & 334 & 337 & 226 & - & - \\
\hline Rate/1000 person years & 1.5 & 1.3 & 1.0 & 0.8 & - & - \\
\hline Age, sex, and area adjusted HR $(95 \% \mathrm{CI}) \dagger$ & 1.00 (ref) & $0.90(0.78$ to 1.04$)$ & 0.83 (0.71 to 0.96$)$ & 0.73 (0.60 to 0.88$)$ & $0.90(0.84$ to 0.96$)$ & $<0.001$ \\
\hline Multivariable adjusted HR $(95 \% \mathrm{Cl}) \neq$ & 1.00 (ref) & 0.97 (0.84 to 1.13$)$ & $0.92(0.79$ to 1.08$)$ & 0.84 (0.70 to 1.02$)$ & $0.96(0.90$ to 1.02$)$ & 0.21 \\
\hline \multicolumn{7}{|l|}{ Cerebrovascular disease mortality } \\
\hline No of deaths & 325 & 256 & 240 & 184 & - & - \\
\hline Rate/1000 person years & 1.1 & 1.0 & 0.7 & 0.6 & - & - \\
\hline Age, sex, and area adjusted HR $(95 \% \mathrm{CI}) \dagger$ & 1.00 (ref) & $0.89(0.76$ to 1.06$)$ & $0.72(0.60$ to 0.86$)$ & $0.66(0.53$ to 0.82$)$ & $0.82(0.76$ to 0.89$)$ & $<0.001$ \\
\hline Multivariable adjusted HR $(95 \% \mathrm{Cl}) \ddagger$ & 1.00 (ref) & 0.97 (0.82 to 1.15$)$ & 0.81 (0.68 to 0.98$)$ & 0.78 (0.63 to 0.97$)$ & 0.89 (0.82 to 0.95$)$ & 0.002 \\
\hline
\end{tabular}

cardiovascular disease, heart disease, and cerebrovascular disease were $10183,4187,2560,1342$, and 1005 , respectively. After adjustment for potential confounding variables, higher food guide scores were found to be associated with lower total mortality; multivariable adjusted hazard ratios (95\% confidence intervals) of total mortality for the lowest through highest fourth scores were 1.00, 0.92 (0.87 to 0.97), 0.88 ( 0.83 to 0.93$)$, and 0.85 ( 0.79 to 0.91$)(\mathrm{P}<0.001$ for trend; table 4). The multivariable adjusted hazard ratio of total mortality for a 10 point increment in the score was 0.93 (0.91 to 0.95$)$. 
For cause specific mortality, cardiovascular disease mortality (particularly cerebrovascular disease mortality) was inversely associated with the food guide score; the multivariable adjusted hazard ratios (95\% confidence intervals) of cardiovascular disease mortality for the lowest through highest fourth category of scores were $1.00,0.97$ (0.87 to 1.07), 0.86 (0.77 to 0.97 ), and 0.84 ( 0.73 to 0.96$)$ ( $\mathrm{P}=0.005$ for trend). The corresponding values for cerebrovascular disease mortality were 1.00, 0.97 (0.82 to 1.15 ), 0.81 (0.68 to 0.98 ), and 0.78 (0.63 to $0.97)(\mathrm{P}=0.002)$. The multivariable adjusted hazard ratio ( $95 \%$ confidence interval) of cardiovascular disease and cerebrovascular disease mortality for a 10 point increment in the food guide score were 0.93 (0.89 to 0.98) and 0.89 (0.82 to 0.95), respectively. There was some evidence (though not quite significant) of an inverse association between the score and cancer mortality (hazard ratio for a 10 point increment in the dietary score $0.96,0.93$ to $1.00 ; \mathrm{P}=0.053$ ). These associations appeared to be linear (fig $\mathrm{C}$ and E, appendix 1). Mortality from heart disease was not associated with the score.

Higher modified food guide scores were associated with a lower risk of total, cardiovascular disease, and cerebrovascular disease mortality (table 5); the multivariable adjusted hazard ratios ( $95 \%$ confidence intervals) of total, cardiovascular disease, and cerebrovascular disease mortality for the highest versus lowest fourth of the dietary score were 0.82 ( 0.77 to 0.88 ; $\mathrm{P}<0.001$ for trend), 0.81 ( 0.71 to $0.93 ; \mathrm{P}=0.001$ for trend), and 0.77 ( 0.62 to $0.96 ; \mathrm{P}=0.001$ for trend), respectively. The modified score was inversely associated with cancer mortality, although the association did not reach significance ( $\mathrm{P}=0.06$ for trend).

Regarding components of the food guide score, scores for vegetable dishes, fish and meat dishes, milk, fruits, and total energy were inversely associated with total mortality (table A, appendix 2). Lower mortality was observed for cardiovascular disease among those with a higher score for vegetable dishes or fruits, for cerebrovascular disease among those with a higher score for fish and meat dishes or fruits, and for heart disease among those with a higher milk score.

We observed relatively large attenuation in associations with the food guide score $(\geq 20 \%)$ after additional adjustment for the fruit score (29.3\%) and vegetable dishes score (29.2\%) for total mortality, fruit score (23.5\%), and vegetable dishes score (20.6\%) for cardiovascular disease mortality, and fish and meat dishes score (23.0\%) and fruit score (22.8\%) for cerebrovascular disease mortality (data not shown in table).

In sensitivity analyses, the observed associations did not change after we excluded total deaths during the first three years of follow-up $(n=1031)$. Exclusion of individuals with a history of diabetes, hypertension, or

\begin{tabular}{|c|c|c|c|c|c|c|}
\hline & \multicolumn{4}{|c|}{ Fourths of score } & \multirow{2}{*}{$\begin{array}{l}10 \text { point } \\
\text { increment }\end{array}$} & \multirow[b]{2}{*}{$\mathrm{P}$ trend* } \\
\hline & 1 & 2 & 3 & 4 & & \\
\hline Mean (SD) score & $38.7(5.2)$ & $49.6(2.3)$ & $57.4(2.3)$ & $66.9(3.9)$ & - & - \\
\hline Person years & 300201 & 285424 & 303951 & 294618 & - & - \\
\hline \multicolumn{7}{|l|}{ Total mortality } \\
\hline No of deaths & 3364 & 2731 & 2260 & 1828 & - & - \\
\hline Rate/1000 person years & 11.2 & 9.6 & 7.4 & 6.2 & - & - \\
\hline Age, sex, and area adjusted $\mathrm{HR}(95 \% \mathrm{Cl}) \dagger$ & 1.00 (ref) & 0.89 (0.84 to 0.93$)$ & $0.76(0.72$ to 0.81$)$ & 0.73 (0.68 to 0.78$)$ & 0.89 (0.87 to 0.91$)$ & $<0.001$ \\
\hline Multivariable adjusted $\mathrm{HR}(95 \% \mathrm{Cl}) \neq$ & 1.00 (ref) & 0.93 (0.89 to 0.98$)$ & 0.84 (0.79 to 0.89 ) & 0.82 (0.77 to 0.88$)$ & 0.93 (0.91 to 0.95$)$ & $<0.001$ \\
\hline \multicolumn{7}{|l|}{ Cancer mortality } \\
\hline No of deaths & 1373 & 1109 & 936 & 769 & - & - \\
\hline Rate/1000 person years & 4.6 & 3.9 & 3.1 & 2.6 & - & - \\
\hline Age, sex, and area adjusted HR $(95 \% \mathrm{Cl}) \dagger$ & 1.00 (ref) & 0.91 (0.84 to 0.99$)$ & $0.82(0.75$ to 0.90$)$ & 0.82 (0.74 to 0.91$)$ & $0.93(0.90$ to 0.96$)$ & $<0.001$ \\
\hline Multivariable adjusted HR $(95 \%$ Cl) & 1.00 (ref) & $0.96(0.89$ to 1.04$)$ & 0.90 (0.82 to 0.99$)$ & 0.92 (0.83 to 1.02$)$ & $0.97(0.93$ to 1.00$)$ & 0.06 \\
\hline \multicolumn{7}{|l|}{ Cardiovascular disease mortality } \\
\hline No of deaths & 813 & 675 & 591 & 481 & - & - \\
\hline Rate/1000 person years & 2.7 & 2.4 & 1.9 & 1.6 & - & - \\
\hline Age, sex, and area adjusted HR $(95 \% \mathrm{Cl}) \dagger$ & 1.00 (ref) & $0.87(0.78$ to 0.96$)$ & 0.76 (0.68 to 0.86$)$ & $0.71(0.62$ to 0.81$)$ & 0.88 (0.84 to 0.92$)$ & $<0.001$ \\
\hline Multivariable adjusted HR $(95 \% \mathrm{Cl}) \neq$ & 1.00 (ref) & 0.93 (0.84 to 1.03$)$ & 0.85 (0.76 to 0.96$)$ & 0.81 (0.71 to 0.93$)$ & 0.93 (0.89 to 0.97$)$ & 0.001 \\
\hline \multicolumn{7}{|l|}{ Heart disease mortality } \\
\hline No of deaths & 433 & 370 & 302 & 237 & - & - \\
\hline Rate/1000 person years & 1.4 & 1.3 & 1.0 & 0.8 & - & - \\
\hline Age, sex, and area adjusted HR (95\% Cl)† & 1.00 (ref) & 0.91 (0.79 to 1.05$)$ & $0.76(0.65$ to 0.89$)$ & 0.70 (0.58 to 0.84$)$ & $0.90(0.85$ to 0.95$)$ & $<0.001$ \\
\hline Multivariable adjusted $\mathrm{HR}(95 \% \mathrm{Cl}) \neq$ & 1.00 (ref) & $0.97(0.84$ to 1.12$)$ & 0.85 (0.72 to 0.99$)$ & 0.79 (0.66 to 0.96$)$ & 0.95 (0.89 to 1.00$)$ & 0.07 \\
\hline \multicolumn{7}{|l|}{ Cerebrovascular disease mortality } \\
\hline No of deaths & 309 & 256 & 247 & 193 & - & - \\
\hline Rate/1000 person years & 1.0 & 0.9 & 0.8 & 0.7 & - & - \\
\hline Age, sex, and area adjusted $\mathrm{HR}(95 \% \mathrm{Cl}) \dagger$ & 1.00 (ref) & 0.83 (0.70 to 0.99$)$ & 0.78 (0.65 to 0.93$)$ & 0.66 (0.54 to 0.82$)$ & 0.84 (0.78 to 0.90$)$ & $<0.001$ \\
\hline Multivariable adjusted HR $(95 \%$ Cl) & 1.00 (ref) & 0.89 (0.76 to 1.06$)$ & 0.88 (0.73 to 1.06$)$ & $0.77(0.62$ to 0.96$)$ & 0.89 (0.83 to 0.95$)$ & 0.001 \\
\hline
\end{tabular}


dyslipidaemia ( $\mathrm{n}=20080)$ strengthened the observed associations with total, cardiovascular disease, and cerebrovascular disease mortalities; the multivariable adjusted hazard ratios (95\% confidence interval) of total, cardiovascular disease, and cerebrovascular disease mortalities for the highest fourth of food guide scores compared with the lowest were 0.82 (0.75 to 0.89 ), 0.76 ( 0.63 to 0.91 ), and 0.61 (0.46 to 0.83 ), respectively, and for the modified score were 0.80 ( 0.73 to 0.87 ), 0.74 (0.61 to 0.89 ), and 0.62 (0.46 to 0.83 ), respectively (all $\mathrm{P}<0.001$ for trend).

\section{Subgroup analyses}

In stratified analyses (table B, appendix 2), we observed an inverse association between the score on the food guide for total mortality in individuals with normal weight $(\mathrm{P}<0.001$ for trend) but not in overweight/obese individuals $(\mathrm{P}=0.53$ for trend), and more pronounced in men who smoked $(\mathrm{P}<0.001$ for trend) than in those who did not $(\mathrm{P}=0.03$ for trend); the differences in these subgroup effects were also significant $(\mathrm{P}<0.001$ for interaction with $\mathrm{BMI}$ and $\mathrm{P}=0.03$ for interaction with smoking). There was no significant difference in association with total mortality according to sex and menopausal status.

We observed a significant inverse trend for cancer mortality in men ( $\mathrm{P}=0.02$ for trend) but not in women $(\mathrm{P}=0.96$ for trend); there was, however, no significant evidence of a difference in these effects between men and women ( $\mathrm{P}=0.11$ for interaction by sex). There was a significant effect in individuals with normal weight ( $\mathrm{P}=0.02$ for trend) but not in overweight/obese individuals $(\mathrm{P}=0.79$ for trend); the difference in these subgroup effects was also significant $(\mathrm{P}=0.003$ for interaction by $\mathrm{BMI})$. As regards cardiovascular disease mortality, we observed a significant inverse trend in individuals with normal weight $(\mathrm{P}=0.001$ for trend) but not in overweight/obese individuals ( $\mathrm{P}=0.87$ for trend); there was, however, no significant evidence of a difference in these subgroup effects $(\mathrm{P}=0.69$ for interaction with $\mathrm{BMI})$. There was a significant inverse association with cardiovascular disease mortality in women $(\mathrm{P}=0.01$ for trend) but not in men ( $\mathrm{P}=0.18$ for trend); there was, however, no significant evidence of a difference in these effects between men and women ( $\mathrm{P}=0.08$ for interaction by sex). After we excluded those with a history of diabetes, hypertension, or dyslipidaemia at baseline, the association in men became significant $(\mathrm{P}=0.004$ for trend).

\section{Discussion}

In this large prospective cohort in Japan, individuals with higher adherence to the Japanese Food Guide Spinning Top had a 15\% lower total mortality rate. This protective association was mainly attributable to a reduction in mortality from cerebrovascular disease. The associations observed with the modified score, with a greater emphasis on fish intake relative to red meat intake, were not significantly different from those with the original score. The present study is one of the few to examine the association between adherence to dietary recommendations or guidelines and mortality in Asian populations.

\section{Findings in relation to other studies}

Our findings agree with those from other studies that reported that higher diet quality scores were associated with reduced total mortality. ${ }^{10-23}$ In China, the Chinese Food Pagoda score assesses the adherence to the Chinese Food Pagoda, which is based on food groups according to the Dietary Guidelines for Chinese. ${ }^{19}$ The concept of the Chinese Food Pagoda score is similar to that of the Japanese Food Guide Spinning Top. In the Shanghai Men's Health Study (n=61239) and the Shanghai Women's Health Study ( $\mathrm{n}=73216$ ), the highest fourth of the Chinese Food Pagoda score was associated with a $33 \%$ and $13 \%$ lower rate of total mortality in men and women, respectively, compared with the lowest fourth. ${ }^{19}$ In the Iowa Women's Health Study ${ }^{18}$ and the Whitehall II cohort, ${ }^{22}$ the highest category of the alternate healthy eating index, which includes components similar to those of the Japanese food guide, predicted $18 \%$ and $25 \%$ lower rate of total mortality, respectively. In the Multiethnic Cohort, ${ }^{23}$ the highest diet quality score, including the healthy eating index-2010 and the alternate healthy eating index-2010, was associated with $22-25 \%$ and $21-22 \%$ lower rate of total mortality in men and women, respectively. This epidemiological evidence, including the present findings, suggests that individuals with higher adherence to country specific dietary recommendations have a lower risk of total mortality.

We found that a higher score on the Japanese Food Guide Spinning Top was associated with a lower rate of total mortality over 15 years of follow-up in both men and women. The score we used in this study was based on the score developed by Oba and colleagues, who found no clear association between the scores and risk of total mortality over seven years of follow-up among men from Japan and a significant inverse association among women. ${ }^{10}$ We have no clear explanation for the observed difference in men between our study and the previous one, ${ }^{10}$ but it might be attributable to the different scoring of vegetable dishes and fruits used in each study. When individuals consumed more than the recommended equivalents of vegetable dishes or fruits, their scores for these foods were deducted in the previous Japanese study. In contrast, we assigned the highest score (10 points) to individuals who consumed more than the recommended amounts of vegetable dishes or fruits. Given that higher intake of vegetables and fruits has been associated with a lower risk of mortality, ${ }^{35}$ the previous study ${ }^{10}$ might have underestimated this association.

In most studies, higher diet quality scores have been consistently and strongly associated with reduced mortality from cardiovascular disease..$^{11-20222336}$ In our study, individuals in the highest fourth of the food guide scores had a 16\% lower rate of mortality from cardiovascular disease compared with those in the lowest fourth. This association was partly explained by intake of vegetable dishes and fruits, which have been linked to a lower risk of cardiovascular disease not only in the present study ${ }^{37}$ but also in numerous other studies. ${ }^{35}$ Likewise, the association with 
mortality from cerebrovascular disease was partly explained by intake of fish and meat dishes. A meta-analysis of 18 prospective studies showed a lower risk of cerebrovascular disease associated with high consumption of fish. ${ }^{38}$ A prospective study in Shanghai showed that higher intake of red meat is associated with a lower risk of mortality from haemorrhagic stroke. ${ }^{39}$ Our findings, together with previous reports, suggest that a dietary pattern of high intake of vegetables and fruits and adequate intake of fish and meat can significantly decrease the risk of mortality from cardiovascular disease in East Asian populations, particularly from cerebrovascular disease. ${ }^{40}$

We found a less clear association between score on the Japanese Food Guide Spinning Top and mortality from cancer in the whole study population, corroborating results of previous studies. ${ }^{1011} 14-1618-20222325$ This could reflect the fact that cancer is a heterogeneous endpoint and that diet is involved in the pathogenesis of certain cancers. ${ }^{41}$ In subgroup analyses, we found a significantly decreasing trend of mortality from cancer with closer adherence to the food guide in men but not in women. This could be due to chance, especially as there was no significant evidence of a difference between men and women. If the finding was genuine, however, it might potentially be explained by sex difference of major forms of cancer, on which diet has differential impact. Specifically, vegetables and/or fruits probably protect against cancers of the lung, stomach, oesophagus, mouth, pharynx, and larynx and milk probably protects against colorectal cancer, whereas there is convincing evidence that red meat and processed meat increase the risk of colorectal cancer. ${ }^{42}$ Of all deaths from cancer in the present cohort, the proportions of deaths from the above mentioned cancers were much higher in men $(60.6 \%)$ than in women (40.4\%). We observed a significant inverse association between the diet score and mortality from cancer in individuals with normal weight but not in overweight/ obese individuals. There was also significant evidence of a difference in effects between normal and overweight/obese groups. Further investigation is required to identify specific groups of individuals who benefit from following the Japanese food guidelines in prevention of cancer.

One concern is that the score on the Japanese Food Guide Spinning Top does not consider the quality of fat, which might have a primary impact on risk of cardiovascular disease, as indicated by a recent randomised controlled trial. ${ }^{26}$ Fish, the major (92\%) component of category of white meat in the present study population, is rich in n-3 polyunsaturated fatty acids, whereas red meat, including beef and pork, contains saturated fatty acids. ${ }^{43}$ We developed a modified score by adding the score for the ratio of intake of white to red meat but found no measurable difference in the association between the two scores. This finding could be explained by the higher consumption of fish ${ }^{445}$ and lower consumption of beef and pork ${ }^{4546}$ among the Japanese population compared with Western populations, maintaining an appropriate balance between white and red meat intakes in terms of cardiovascular health. In our study, a higher intake of fish and n-3 polyunsaturated fatty acids was associated with a decreased risk of myocardial infarction, ${ }^{47}$ and a higher intake of saturated fatty acids was associated with a decreased risk of stroke. ${ }^{48}$

\section{Strengths and limitations}

The strengths of the present study were its population based prospective design involving a large cohort, the long duration of follow-up (15 years), and the use of a validated food frequency questionnaire. Our study also has several limitations. Firstly, the food frequency questionnaire was not developed for the purpose of estimating the adherence to the Japanese Food Guide Spinning Top. However, we used 125 of 147 (85\%) food and beverage items in the validated food frequency questionnaire, and we retrospectively calculated dish servings according to the food guide. Thus, well designed food frequency questionnaires could be used to estimate dish servings and calculate the adherence score. Secondly, dietary intake was assessed only at baseline and might not represent long term habitual intake relevant to mortality. In the present cohort, however, dietary intake was generally stable over time; the Spearman rank correlation coefficients of intake of each dish category between the second and the third surveys ranged between 0.46 and 0.64 in men and between 0.45 and 0.64 in women. Thirdly, our analysis included individuals with a history of diabetes, hypertension, or dyslipidaemia, who might have received advice to follow the dietary guidelines for each disease and changed their diet accordingly. In sensitivity analyses, the associations between the dietary score and mortality were strengthened after we excluded these individuals. Finally, we cannot completely rule out the effects of confounding by residual and unmeasured variables. One such example is smoking, which is a strong predictor of mortality ${ }^{49}$ and is associated with dietary habits. In stratified analysis, we found more pronounced inverse associations between dietary scores and mortality in men who smoked; however, this observation might also be a result of incomplete control of smoking intensity. Furthermore, the observed associations were materially unchanged after adjustment for the number of cigarettes smoked, suggesting that smoking intensity is unlikely to have contributed to residual confounding in the present study.

\section{Conclusion}

In summary, individuals with closer adherence to the Japanese dietary guidelines had a lower risk of death from all causes and cardiovascular disease, particularly cerebrovascular disease. Our findings suggest that balanced consumption of energy, grains, vegetables, fruits, meat, fish, eggs, soy products, dairy products, confectionaries, and alcoholic beverages can contribute to longevity by decreasing the risk of death, predominantly from cardiovascular disease, in the Japanese population.

We are indebted to the Aomori, Iwate, Ibaraki, Niigata, Osaka, Kochi, Nagasaki, and Okinawa Cancer Registries for providing their incidence data. 


\section{Members of the Japan Public Health Center based Prospective (JPHC) Study Group}

S Tsugane (principal investigator), N Sawada, M Iwasaki, S Sasazuki, T Yamaji, T Shimazu, T Hanaoka, National Cancer Centre, Tokyo; । Ogata, S Baba, T Mannami, A Okayama, Y Kokubo, National Cerebral and Cardiovascular Centre, Osaka; K Miyakawa, F Saito, A Koizumi, Y Sano, I Hashimoto, T Ikuta, Y Tanaba, H Sato, Y Roppongi, T Takashima, H Suzuki, Iwate Prefectural Ninohe Public Health Centre, Iwate; Y Miyajima, N Suzuki, S Nagasawa, Y Furusugi, N Nagai, Y Ito, S Komatsu, T Minamizono, Akita Prefectural Yokote Public Health Centre, Akita; H Sanada, Y Hatayama, F Kobayashi, H Uchino, Y Shirai, T Kondo, R Sasaki, Y Watanabe, Y Miyagawa, Y Kobayashi, M Machida, K Kobayashi, M Tsukada, Nagano Prefectural Saku Public Health Centre, Nagano; Y Kishimoto, E Takara, T Fukuyama, M Kinjo, M Irei, H Sakiyama, Okinawa Prefectural Chubu Public Health Centre, Okinawa; K Imoto, H Yazawa, T Seo, A Seiko, F Ito, F Shoji, R Saito, Katsushika Public Health Centre, Tokyo; A Murata, K Minato, K Motegi, T Fujieda, S Yamato, Ibaraki Prefectural Mito Public Health Centre, Ibaraki; K Matsui, T Abe, M Katagiri, M Suzuki, K Matsui, Niigata Prefectural Kashiwazaki and Nagaoka Public Health Centre, Niigata; M Doi, A Terao, Y Ishikawa, T Tagami, Kochi Prefectural Chuo-higashi Public Health Centre, Kochi; H Sueta, H Doi, M Urata, N Okamoto, F Ide, H Goto, R Fujita, Nagasaki Prefectural Kamigoto Public Health Center, Nagasaki; H Sakiyama, N Onga, H Takaesu, M Uehara, T Nakasone, M Yamakawa, Okinawa Prefectural Miyako Public Health Centre, Okinawa; F Horii, I Asano, H Yamaguchi, K Aoki, S Maruyama, M Ichii, M Takano, Osaka Prefectural Suita Public Health Centre, Osaka; Y Tsubono, Tohoku University, Miyagi; K Suzuki, Research Institute for Brain and Blood Vessels Akita, Akita; Y Honda, K Yamagishi, S Sakurai, N Tsuchiya, University of Tsukuba, Ibaraki; M Kabuto, National Institute for Environmental Studies, Ibaraki; M Yamaguchi, Y Matsumura, S Sasaki, S Watanabe, National Institute of Health and Nutrition, Tokyo; M Akabane, Tokyo University of Agriculture, Tokyo; T Kadowaki, M Inoue, University of Tokyo, Tokyo, M Noda, T Mizoue, National Centre for Global Health and Medicine, Tokyo; Y Kawaguchi, Tokyo Medical and Dental University, Tokyo; Y Takashima, Y Yoshida, Kyorin University, Tokyo; K Nakamura, R Takachi, Niigata University, Niigata; J Ishihara, Sagami Women's University, Kanagawa; S Matsushima, S Natsukawa, Saku General Hospital, Nagano; H Shimizu, Sakihae Institute, Gifu; H Sugimura, Hamamatsu University School of Medicine, Shizuoka; S Tominaga, Aichi Cancer Centre, Aichi; N Hamajima, Nagoya University, Aichi; H Iso, T Sobue, Osaka University, Osaka; M lida, W Ajiki, A loka, Osaka Medical Centre for Cancer and Cardiovascular Disease, Osaka; S Sato, Chiba Prefectural Institute of Public Health, Chiba; E Maruyama, Kobe University, Hyogo; M Konishi, K Okada, I Saito, Ehime University, Ehime; N Yasuda, Kochi University, Kochi; S Kono, Kyushu University, Fukuoka; S Akiba, Kagoshima University, Kagoshima; T Isobe, Keio University, Tokyo; Y Sato, Tokyo Gakugei University, Tokyo.

Contributors: ST was involved in the design of study as the principal investigator; ST, NS, and SS conducted the survey; KK, SA, IK, AG, TM, and $M N$ drafted the plan for the data analyses; KK, SA, and AG conducted data analysis; $\mathrm{AG}$ and TM provided statistical expertise; KK drafted the manuscript; KK and TM had primary responsibility for final content; and all authors were involved in interpretation of the results and revision of the manuscript and approved the final version of the manuscripts. KK and TM are guarantors.

Funding: This study was supported by National Cancer Centre research and development fund (23-A-31[toku] and 26-A-2) (since 2011), a grant-in-aid for Cancer Research from the Ministry of Health, Labour and Welfare of Japan (from 1989 to 2010), Practical Research Project for Life-Style related Diseases including Cardiovascular Diseases and Diabetes Mellitus (15ek0210021 h0002) from the Japan Agency for Medical Research and Development, JSPS KAKENHI Grant No $15 \mathrm{HO} 4779$, and grants-in-aid for research from the National Centre for Global Health and Medicine (26A-201).

Competing interests: All authors have completed the ICMJE uniform disclosure form at www.icmje.org/coi_disclosure.pdf and declare: no support from any organisation for the submitted work; no financial relationships with any organisations that might have an interest in the submitted work in the previous three years; no other relationships or activities that could appear to have influenced the submitted work.

Ethical approval: This study was approved by the institutional review board of the National Cancer Centre of Japan and the ethics committee of the National Centre for Global Health and Medicine, Japan. Although we did not require written informed consent, the study participants were informed of the objectives of the study; participants who responded to the questionnaire survey were considered to have consented to participating in the survey.
Transparency: KK and TM affirm that the manuscript is an honest, accurate, and transparent account of the study being reported; that no important aspects of the study have been omitted; and that any discrepancies from the study as planned have been explained. Data sharing: No additional data available.

This is an Open Access article distributed in accordance with the Creative Commons Attribution Non Commercial (CC BY-NC 3.0) license, which permits others to distribute, remix, adapt, build upon this work non-commercially, and license their derivative works on different terms, provided the original work is properly cited and the use is noncommercial. See: http://creativecommons.org/licenses/by-nc/3.0/.

1 World Health Organization. World Health Statistics 2014. WHO, 2015. http://apps.who.int/iris/bitstream/10665/112738/1/9789240692671 eng.pdf?ua=1.

2 Ikeda N, Saito E, Kondo N, et al. What has made the population of Japan healthy? Lancet 2011;378:1094-105. doi:10.1016/ S0140-6736(11)61055-6.

3 Tominaga S, Kuroishi T. An ecological study on diet/nutrition and cancer in Japan. Int J Cancer 1997;Suppl 10:2-6. doi:10.1002/ (SICI) 1097-0215(1997)10+<2::AID-IJC2>3.0.CO;2-C.

4 Ohno Y. Health development in Japan: determinants, implications and perspectives. World Health Stat Q 1985;38:176-92.

5 National Institute of Health and Nutrition. The National Health and Nutrition Survey 1975-2002. 2005. http://www0.nih.go.jp/eiken/ chosa/kokumin_eiyou/doc theme/tbl_1100.xls.

6 Wirt A, Collins CE. Diet quality--what is it and does it matter?Public Health Nutr 2009;12:2473-92. doi:10.1017/S136898000900531X.

7 Kennedy ET, Ohls J, Carlson S, Fleming K. The Healthy Eating Index: design and applications. J Am Diet Assoc 1995;95:1103-8. doi:10.1016/S0002-8223(95)00300-2

8 McCullough ML, Feskanich D, Stampfer MJ, et al. Diet quality and major chronic disease risk in men and women: moving toward improved dietary guidance. Am J Clin Nutr 2002;76:1261-71.

9 Yoshiike N, Hayashi F, Takemi Y, Mizoguchi K, Seino F. A new food guide in Japan: the Japanese food guide Spinning Top. Nutr Rev 2007;65:149-54. doi:10.1301/nr.2007.apr.149-154.

10 Oba S, Nagata C, Nakamura K, et al. Diet based on the Japanese Food Guide Spinning Top and subsequent mortality among men and women in a general Japanese population. J Am Diet Assoc 2009;109:1540-7. doi:10.1016/j.jada.2009.06.367.

11 Trichopoulou A, Costacou T, Bamia C, Trichopoulos D. Adherence to a Mediterranean diet and survival in a Greek population. N Engl/ Med 2003;348:2599-608. doi:10.1056/NEJMoa025039.

12 Trichopoulou A, Kouris-Blazos A, Wahlqvist ML, et al. Diet and overall survival in elderly people. BMJ 1995;311:1457-60. doi:10.1136 bmi.311.7018.1457.

13 Osler M, Schroll M. Diet and mortality in a cohort of elderly people in a north European community. Int J Epidemiol 1997;26:155-9. doi:10.1093/ije/26.1.155

14 Kant AK, Schatzkin A, Graubard BI, Schairer C. A prospective study of diet quality and mortality in women. JAMA 2000;283:2109-15. doi:10.1001/jama.283.16.2109.

15 Mai V, Kant AK, Flood A, Lacey JV Jr, Schairer C, Schatzkin A. Diet quality and subsequent cancer incidence and mortality in a prospective cohort of women. Int J Epidemiol 2005;34:54-60. doi:10.1093/ije/dyh388.

16 Michels KB, Wolk A. A prospective study of variety of healthy foods and mortality in women. Int J Epidemiol 2002;31:847-54. doi:10.1093/ ije/31.4.847.

17 Knoops KT, Groot de LC, Fidanza F, Alberti-Fidanza A, Kromhout D, van Staveren WA. Comparison of three different dietary scores in relation to 10-year mortality in elderly European subjects: the HALE project. Eur / Clin Nutr 2006:60:746-55. doi:10.1038/ sj.ejcn.1602378.

18 Mursu J, Steffen LM, Meyer KA, Duprez D, Jacobs DR Jr. Diet quality indexes and mortality in postmenopausal women: the lowa Women’s Health Study. Am J Clin Nutr 2013;98:444-53. doi:10.3945/ajcn.112.055681

19 Yu D, Zhang X, Xiang YB, et al. Adherence to dietary guidelines and mortality: a report from prospective cohort studies of 134,000 Chinese adults in urban Shanghai. Am J Clin Nutr 2014;100:693-700. doi:10.3945/ajcn.113.079194

20 Seymour ID, Calle EE, Flagg EW, Coates RI, Ford ES, Thun MJ. American Cancer Society. Diet quality index as a predictor of short-term mortality in the American Cancer Society Cancer Prevention Study II Nutrition Cohort. Am J Epidemiol 2003;157:980-8. doi:10.1093/aje/ kwg077.

21 Bazelmans C, De Henauw S, Matthys C, et al. Healthy food and nutrient index and all cause mortality. Eur J Epidemiol 2006;21:14552. doi:10.1007/s10654-005-5699-8.

22 Akbaraly TN, Ferrie JE, Berr C, et al. Alternative Healthy Eating Index and mortality over 18 y of follow-up: results from the Whitehall II cohort. Am J Clin Nutr 2011;94:247-53. doi:10.3945/ ajcn.111.013128. 
23 Harmon BE, Boushey C), Shvetsov YB, et al. Associations of key diet-quality indexes with mortality in the Multiethnic Cohort: the Dietary Patterns Methods Project. Am / Clin Nutr 2015;101:587-97. doi:10.3945/ajcn.114.090688.

24 Atkins JL, Whincup PH, Morris RW, Lennon LT, Papacosta O, Wannamethee SG. High diet quality is associated with a lower risk of cardiovascular disease and all-cause mortality in older men. J Nutr 2014;144:673-80. doi:10.3945/jn.113.186486.

25 Lagiou P, Trichopoulos D, Sandin S, et al. Mediterranean dietary pattern and mortality among young women: a cohort study in Sweden. BrJ Nutr 2006;96:384-92. doi:10.1079/BJN20061824.

26 Reidlinger DP, Darzi J, Hall WL, Seed PT, Chowienczyk PJ, Sanders TA. Cardiovascular disease risk REduction Study (CRESSIDA) investigators. How effective are current dietary guidelines for cardiovascular disease prevention in healthy middle-aged and older men and women? A randomized controlled trial. Am J Clin Nutr 2015:101:922-30 doi:10.3945/ajcn.114.097352.

27 Tsugane S, Sawada N. The JPHC study: design and some findings on the typical Japanese diet. Jpn J Clin Oncol 2014;44:777-82. doi:10.1093/ijco/hyu096.

28 Sasaki S, Kobayashi M, Ishihara J, Tsugane S. JPHC. Self-administered food frequency questionnaire used in the 5-year follow-up survey of the JPHC Study: questionnaire structure, computation algorithms, and area-based mean intake. J Epidemiol 2003;13(Suppl):S13-22. doi:10.2188/jea.13.1sup_13.

29 Science and Technology Agency. 5th revised and enlarged. Printing Bureau of the Ministry of Finance, 2005. [Standard tables of food composition in Japan]

30 Ishihara J, Sobue T, Yamamoto S, et al. JPHC. Validity and reproducibility of a self-administered food frequency questionnaire in the JPHC Study Cohort II: study design, participant profile and results in comparison with Cohort I. J Epidemiol 2003;13(Suppl):S134-47. doi:10.2188/jea.13.1sup_134.

31 Sasaki S, Ishihara J, Tsugane S. JPHC. Reproducibility of a self-administered food frequency questionnaire used in the 5-year follow-up survey of the JPHC Study Cohort I to assess food and nutrient intake. J Epidemiol 2003:13(Suppl):S115-24. doi:10.2188/jea.13.1sup 115.

32 Sasaki S, Kobayashi M, Tsugane S. JPHC. Validity of a selfadministered food frequency questionnaire used in the 5-year follow-up survey of the JPHC Study Cohort I: comparison with dietary records for food groups. J Epidemiol 2003;13(Suppl):S57-63. doi:10.2188/jea.13.1sup_57.

33 World Health Organization. ICD-10: international statistical classification of diseases and related health problems: 10th revision. World Health Organization, 1992.

34 Rubin DB. Multiple imputation for nonresponse in surveys. Wiley, 1987. doi:10.1002/9780470316696.

35 Wang X, Ouyang Y, Liu J, et al. Fruit and vegetable consumption and mortality from all causes, cardiovascular disease, and cancer: systematic review and dose-response meta-analysis of prospective cohort studies. BM/ 2014;349:94490. doi:10.1136/ bmj.g4490.
36 Sofi F, Abbate R, Gensini GF, Casini A. Accruing evidence on benefits of adherence to the Mediterranean diet on health: an updated systematic review and meta-analysis. Am I Clin Nutr 2010;92:1189 96. doi:10.3945/ajen.2010.29673

37 Takachi R, Inoue M, Ishihara J, et al. JPHC Study Group. Fruit and vegetable intake and risk of total cancer and cardiovascular disease: Japan Public Health Center-Based Prospective Study. Am J Epidemiol 2008;167:59-70. doi:10.1093/aje/kwm263.

38 Chowdhury R, Stevens S, Gorman D, et al. Association between fish consumption, long chain omega 3 fatty acids, and risk of cerebrovascular disease: systematic review and meta-analysis. $B M$ 2012;345:e6698. doi:10.1136/bmj.e6698.

39 Takata Y, Shu XO, Gao YT et al. Red meat and poultry intakes and risk of total and cause-specific mortality: results from cohort studies of Chinese adults in Shanghai. PLoS One 2013;8:e56963. doi:10.1371/ journal.pone.0056963.

40 Hata J, Kiyohara Y. Epidemiology of stroke and coronary artery disease in Asia. Circ/ 2013;77:1923-32. doi:10.1253/circj.CJ-13-0786

41 Chiuve SE, Fung TT, Rimm EB, et al. Alternative dietary indices both strongly predict risk of chronic disease. / Nutr 2012;142:1009-18. doi:10.3945/jn.111.157222

42 World Cancer Research Fund, American Institute for Cancer Research. Food, nutrition, physical activity, and the prevention of cancer: a global perspective.AICR, 2007.

43 Science and Technology Agency. [Standard tables of food composition in Japan, fatty acids section.] 5th revised and enlarged ed. Tokyo, Japan: Printing Bureau of the Ministry of Finance, 2005.

44 FAOSTAT. Fish, Seafood. Food and agriculture organization of the United Nations statistics division, 2013. http://faostat.fao.org/.

45 Kenko Eiyo Joho Kenkyukai. The National Health and Nutrition Survey in Japan, 2010.Daiichi-shuppan, 2013.

46 Daniel CR, Cross AJ, Koebnick C, Sinha R. Trends in meat consumption in the USA. Public Health Nutr 2011;14:575-83. doi:10.1017/ S1368980010002077.

47 Iso H, Kobayashi M, Ishihara J, et al. JPHC Study Group. Intake of fish and $\mathrm{n} 3$ fatty acids and risk of coronary heart disease among Japanese: the Japan Public Health Center-Based (JPHC) Study Cohort I. Circulation 2006;113:195-202. doi:10.1161/CIRCULATIONAHA.105.581355.

48 Yamagishi K, Iso H, Kokubo Y, et al. JPHC Study Group. Dietary intake of saturated fatty acids and incident stroke and coronary heart disease in Japanese communities: the JPHC Study. Eur Heart J 2013;34:1225-32. doi:10.1093/eurheartj/eht043.

49 Hara M, Sobue T, Sasaki S, Tsugane S. Smoking and risk of premature death among middle-aged Japanese: ten-year follow-up of the Japan Public Health Center-based prospective study on cancer and cardiovascular diseases (JPHC Study) cohort I. Jpn J Cancer Res 2002;93:6-14. doi:10.1111/j.1349-7006.2002.tb01194.x.

Appendix 1: Supplementary figures
Appendix 2: Supplementary tables 